\section{Endovascular Recanalization of a Hepatic Vein in Budd-Chiari Syndrome: A Collateral Loop-Guided Approach}

From: Marta Baptista, MBBS

Luisa Costa Andrade, MD

Emanuel Furtado, MD

Paulo Donato, MD, PhD

From the Imaging Department (M.B.)

Coimbra University Hospital

Rua Fonseca Pinto

3000-075 Coimbra, Portugal;

Imaging Department and Medical School (L.C.A., P.D.) and

Hepatic Transplantation Department (E.F.)

Centro Hospital e Universitario de Coimbra

Coimbra, Portugal

\section{Editor:}

This report was granted exemption from institutional review board approval. The authors report a case of endovascular recanalization and stent placement in the right hepatic vein (HV) by using a collateral loop-guided approach through a caudate-lobe patent accessory HV.

A 9-year-old boy presented with a 2-month history of abdominal distension, left thigh pain, and claudication. Blood tests revealed erithrocytosis, $\gamma$-glutamyl transpeptidase level of $143 \mathrm{U} / \mathrm{L}$, and lactate level of $2,30 \mathrm{mmol} / \mathrm{L}$. Coagulation, thrombophilia studies, and copper levels were normal. Autoantibody screens were negative, and autoimmune lymphoproliferative syndrome was excluded by flow cytometry. Genetic analysis showed a nonspecific $J A K 2$ gene polymorphism.

Doppler ultrasound (US) demonstrated narrowed HVs with decreased flow $(10 \mathrm{~cm} / \mathrm{s})$, and thrombosis of the confluence of the right, middle, and left HVs (Fig 1). A tortuous caudate lobe vein was identified. The portal vein had antegrade flow with normal velocity $(33 \mathrm{~cm} / \mathrm{s})$. The hepatic artery had normal resistive indices. Hepatomegaly (liver length at the midclavicular line of $15 \mathrm{~cm}$ ) and ascites were noted. Computed tomographic (CT) angiography confirmed the HV obstruction and demonstrated caudate lobe hypertrophy with retrohepatic vena cava compression $(3 \mathrm{~mm})$. The caudatelobe accessory HV draining into the inferior vena cava (IVC) was identified (Fig 2).

The patient was referred to our institution for angiographic evaluation and, if possible, retrograde recanalization of the HVs. The venography and endovascular treatment were performed through a right transjugular approach. A 6-F, 11-cm-long sheath introducer (Boston Scientific, Natick, Massachusetts) was inserted into the right jugular vein, through which a $65-\mathrm{cm}-$ long, 5-F multipurpose 0.038-inch catheter (Cordis, Warren,

None of the authors have identified a conflict of interest.

http://dx.doi.org/10.1016/j.jvir.2014.07.023
New Jersey) was advanced into the abdominal IVC. It was impossible to selectively cannulate the main HVs because of the confluence obstruction. The accessory HV draining the caudate lobe directly into the IVC was cannulated. A venous shunt between the accessory caudate lobe vein and the right $\mathrm{HV}$ was found. A roadmap image of this shunt was obtained (Fig 3). A 180-cm-long, 0.035-inch nitinol guide wire (Terumo, Tokyo, Japan) was advanced through the shunt into the right HV. Venography at this time revealed a $4-\mathrm{cm}$ obstruction of the right $\mathrm{HV}$. It was possible to cross the obstructing clot and reach the IVC with the Terumo guide wire. The 6-F introducer was replaced by an 11-cm-long, 8-F sheath introducer (Boston Scientific), which allowed a loop fashioned from a 300-cm-long, 0.014-inch Cougar LS Nitinol guide wire (Meditronic,

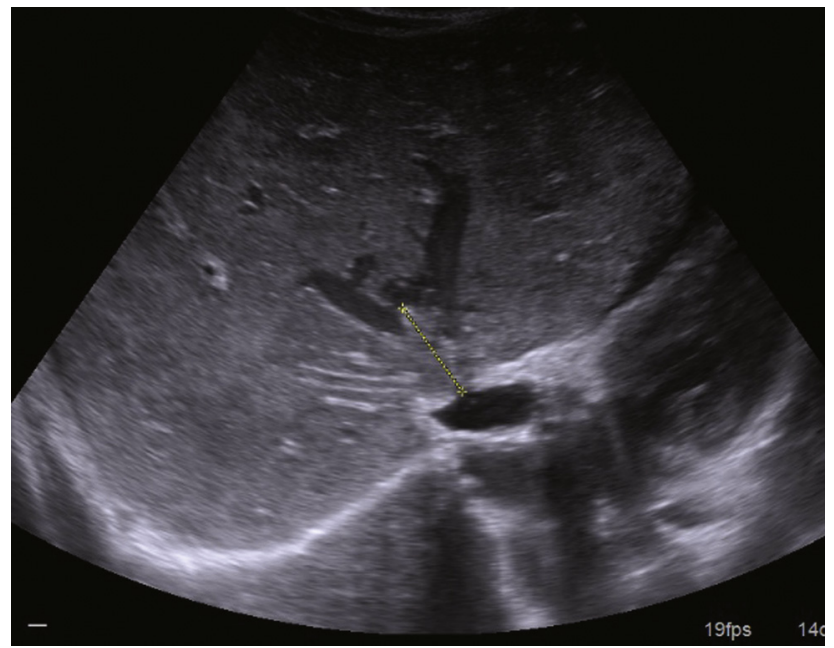

Figure 1. US scan shows echoic material (between calipers) at the confluence of the left and middle HVs, suggestive of obstructing clot. (Available in color online at www.jvir.org.)

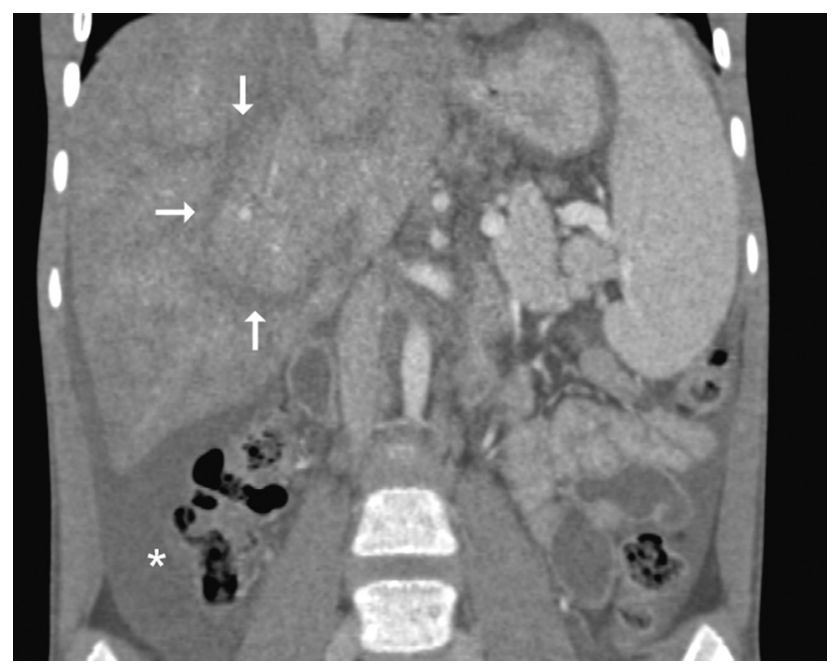

Figure 2. Postcontrast coronal CT image shows unenhacing caudate-lobe HV communicating with the unopacified main HV through an intrahepatic collateral vessel (arrows). Note the presence of ascites (asterisk). 
Minneapolis, Minnesota) to be pushed through the jugular access. This was used to snare the Terumo wire extremity within the IVC. The looped Cougar LS wire was pulled through the jugular venous access, and the floppy end of the Terumo wire was exteriorized (Fig 4a). An Absolute Pro self-expanding vascular nitinol stent $(8.0 \mathrm{~mm}$ in diameter and $40 \mathrm{~mm}$ in length; Abbott, Abbott Park, Illinois) on an $80-\mathrm{cm}$ sheath was advanced over the floppy end of the Terumo wire and deployed within the right HV (Fig 4b). A Charger balloon (7.0 mm in diameter and $40 \mathrm{~mm}$ in length; Boston Scientific) was

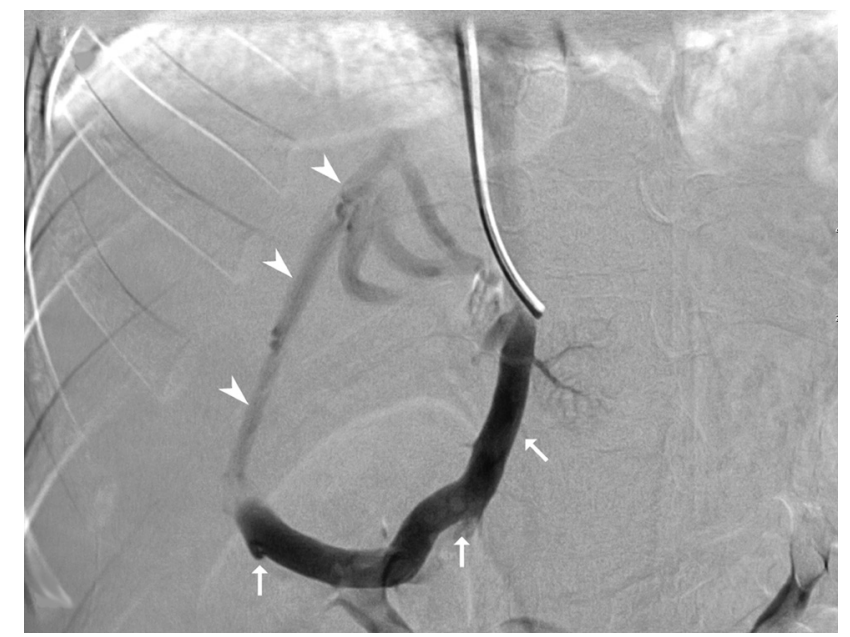

Figure 3. Venographic roadmap of the caudate lobe HV (arrows) and collateral vessel between the caudate-lobe HV and right HV (arrowheads). used to fully expand the stent, after which stent patency was confirmed. During the procedure, the patient received low molecular weight heparin (3,000 IU), and, the following day, long-term anticoagulation was commenced for 1 year.

US evaluation 4 days later revealed a decrease in hepatic size (liver length at the midclavicular line of 14 $\mathrm{cm})$. Doppler US showed turbulent hepatofugal flow within the stent (Fig 5), with velocities reaching $130 \mathrm{~cm} / \mathrm{s}$. The portal vein maintained antegrade flow and normal velocity $(50 \mathrm{~cm} / \mathrm{s})$ and the ascites had resolved.

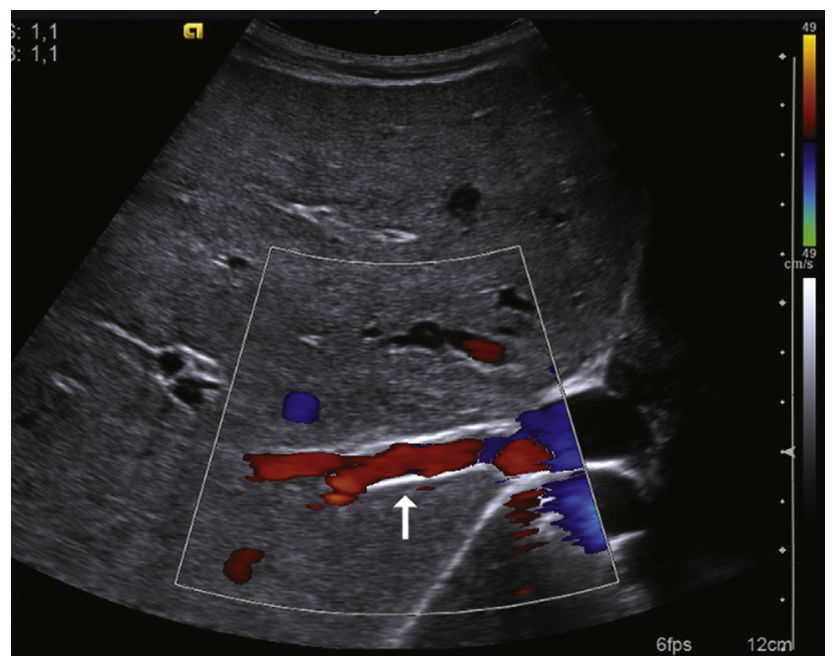

Figure 5. Doppler US image demonstrates stent (arrow) patency within the right HV 4 days after treatment. (Available in color online at www.jvir.org.)

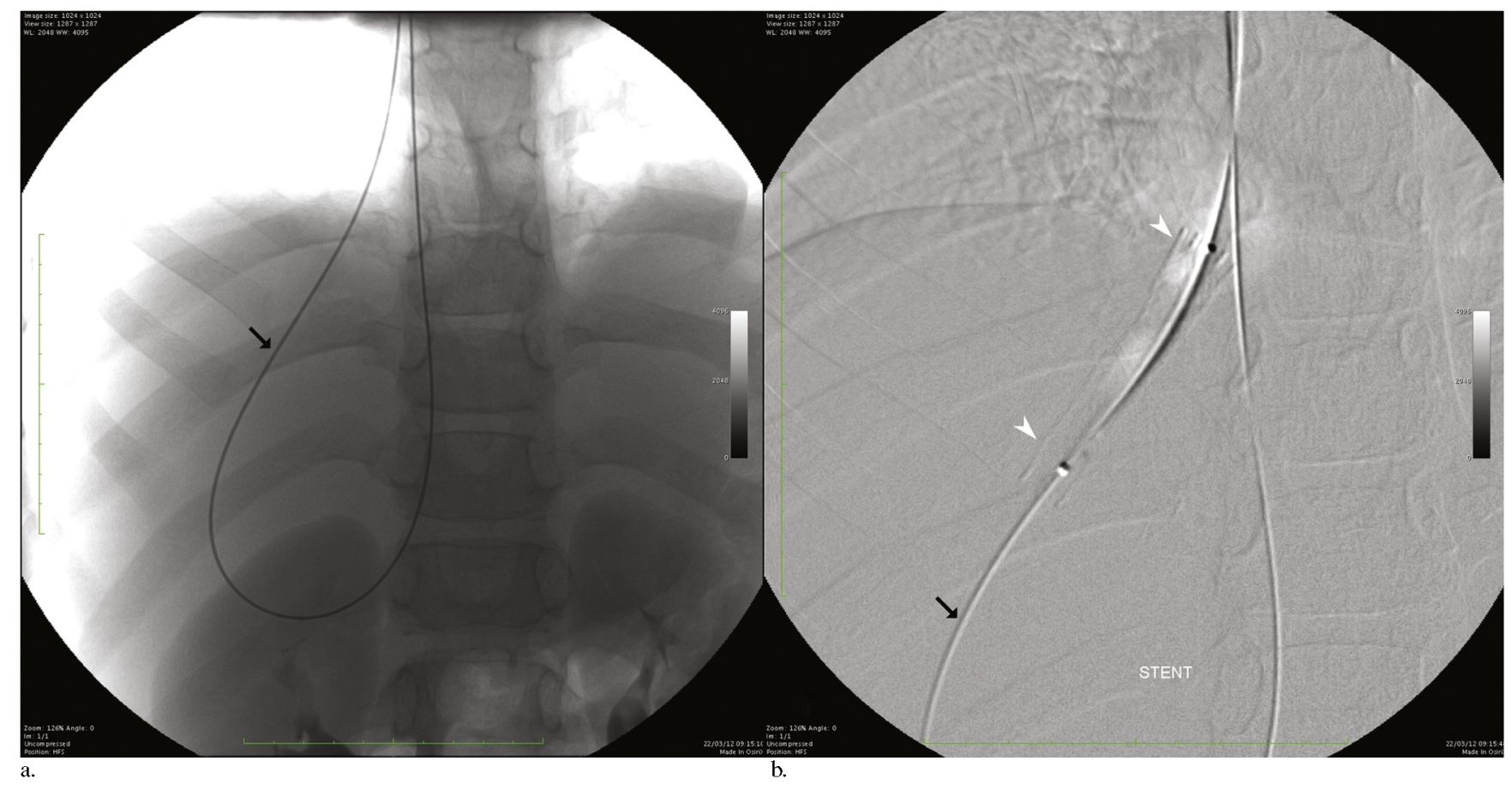

Figure 4. (a) Radiograph shows a Terumo guide wire loop (arrow) from the IVC through the venous shunt and back into the IVC. (b) Digital subtraction image shows a stent (arrowheads) on the Terumo guide wire floppy end arm (arrow). (Available in color online at www.jvir.org.) 
Throughout the following year, Doppler US follow-up did not show evidence of stent thrombosis, and there was clinical improvement (increase in weight of $7 \mathrm{~kg}$ and normal development, but a $\gamma$-glutamyl transpeptidase level of $140 \mathrm{U} / \mathrm{L}$ ).

Endovascular recanalization of the HVs used to be done with balloon angioplasty, but it has been shown that stent implantation decreases the restenosis rate to $7 \%$ regardless of the obstruction etiology (1); stent placement is therefore now common practice.

Zhang et al (2), who studied the long-term effect of stent placement in Budd-Chiari syndrome, obtained an overall HV stent patency rate of $90.9 \%$. The risk of stent occlusion was higher in HV stents (9.1\%) than in IVC stents (3.3\%).

Antegrade percutaneous approaches have been described, but present a potentially increased hemorrhagic risk as the liver capsule is punctured (2). Even though it is probably safer, our approach cannot be widely used because direct drainage of the caudate lobe into the IVC is not always present. However, if this phenomenon is noted, we believe venography of this vessel should be performed to identify possible shunts. It is recognized that the caudate-lobe $\mathrm{HV}$ is engorged in $50 \%$ of patients with Budd-Chiari syndrome, and serves a pivotal role in liver drainage through multiple collateral vessels (3).

In summary, this report presents a case of a successful percutaneous endovascular recanalization, stent implantation, and balloon-aided stent expansion in the right HV in Budd-Chiari syndrome achieved through a collateral approach in a patient with a shunt between a patent caudate accessory HV and right HV.

\section{REFERENCES}

1. Ferral H, Behrens G, Lopera J. Budd-Chiari syndrome. AJR Am J Roentgenol 2012; 199:737-745.

2. Zhang $C, F u L, X u L$, Zhang G, et al. Long-term effect of stent placement in 115 patients with Budd-Chiari syndrome. World J Gastroenterol 2003; 9: 2587-2591

3. Bargalló X, Gilatert R, Nicolau C, et al. Sonography of Budd-Chiari syndrome. AJR Am J Roentgenol 2006; 187:W33-W42.

\section{Use of PhaSeal Transfer System during Chemoembolization to Prevent Unintentional Leakage of Chemotherapeutic Agents during Preparation and Delivery}

From: Sohail Contractor, MD, FSIR

Abhishek Kumar, MD

Michelle Phillip, BS

Dhruv Patel, BA

Jenicka Karcich, BS

Piotr Kisza, MD

None of the authors have identified a conflict of interest.

http://dx.doi.org/10.1016/j.jvir.2014.09.006
Department of Radiology, Section of Interventional Radiology (S.C., M.P., D.P., J.K., P.K.)

Rutgers-New Jersey Medical School

150 Bergen Street, UH C 318 A

Newark, NJ 07101-1709

Section of Interventional Radiology (A.K.)

Mount Sinai Beth Israel Medical Center

New York, New York

\section{Editor:}

This report meets criteria for institutional review board exemption at our institute. Transarterial chemoembolization is a common procedure for the management of primary and secondary hepatic malignancies. The traditional preparation process of the chemoembolic suspension involves mixing the chemotherapeutic agents (usually doxorubicin, mitomycin, or cisplatin or a combination) with ethiodized oil (Lipiodol; Guerbet LLC, Bloomington, Indiana) across a three-way open transfer device (OTD) (1). However, the high viscosity of the agents tends to degrade the material in the OTD and the syringes and can lead to hazardous leakage of the chemoembolic suspension onto the procedure field posing a risk to the operator and other staff in the procedure area. The National Institute of Occupational Safety and Health, the American Society of Health-System Pharmacists, and the International Society of Oncology Pharmacy Practitioners have published alerts and guidelines regarding the safe handling of chemotherapeutic and other potentially vesicant drugs (2).

A closed-system drug transfer system (CSDTS) that would eliminate this risk to operators is desirable. Various CSDTS exist, such as ChemoLock (ICU Medical, San Clemente, California) and OnGuard (B. Braun Medical, Inc, Bethlehem, Pennsylvania); however, their use in interventional radiology procedures has not been described to our knowledge. One CSDTS, the PhaSeal system (Becton, Dickinson and Company, Dublin, Ireland), is designed to ensure leak-free transfer of hazardous material that meets National Institute of Occupational Safety and Health guidelines, and its use has been reported by pharmacies to prepare chemotherapeutic and other hazardous vesicant agents (2). In our practice, we often faced leakage of chemotherapeutic agents while mixing with ethiodized oil or injecting the suspension secondary to "melt-down" of the plastic OTD. We reviewed our practice with the Pharmacy and Therapeutics committee at our hospital who recommended our use of the PhaSeal system. This system had been used by our hospital pharmacy for the past 4 years. Consequently, we introduced the PhaSeal transfer system to replace the three-way OTD.

We present our initial experience using this system as a replacement for the three-way OTD. The device consists of the PhaSeal Injector Luer Lock and Connector Luer Lock components and a fluid-dispensing connector 